\title{
HARDCORE EN PRIMERA PERSONA. LA VISTA SUBJETIVA DEL VIDEOJUEGO EN EL CINE
}

\author{
Mario-Paul MARTÍNEZ
}

$\mathrm{UHM}$

\section{RESUMEN}

El empleo de la cámara subjetiva no es un fenómeno nuevo en el cine. Sin embargo, y a pesar de tratarse de una técnica recurrente en la cinematografía, son escasas las películas filmadas enteramente bajo este recurso. Desde su primera incursión en 1947, La dama del lago (Robert Montgomery), apenas se cuenta una decena de ejemplos, y la mayoría se agrupan en los últimos años, cuando la tecnología y los formatos digitales han dado un nuevo impulso a esta peculiar mirada.

Entre ellos, Hardcore Henry (Ilya Naishuller, 2016), un film en vista subjetiva que aprovecha una de las perspectivas imperantes de la actualidad: la de los videojuegos. Una película esencial para estudiar el legado de La dama del lago bajo las claves del cine espectáculo, y para explorar, también, las interacciones del cine con un universo lúdico que, como a nadie se le escapa, el espectador cada vez conoce mejor. A través de éste, y otros ejemplos conectados como Doom (Andrzej Bartkowiak, 2005), Gamer (Neveldine y Taylor, 2009) o Elephant (Gus Van Sant, 2003), se busca aportar luz a una de las preguntas clave de la cinematografía actual: ¿Qué ocurre cuando decides filmar una película desde la perspectiva de un videojuego?

Palabras clave: Cine, Videojuego, POV, Cámara Subjetiva, espectáculo.

\section{ABSTRACT}

The use of the subjective camera is not a new phenomenon in the cinema. However, and despite being a recurring technique in cinematography, the films filmed entirely under this feature are few. Since her first foray in 1947, The Lady of the Lake (Robert Montgomery), there are scarcely a dozen examples, and most are grouped in recent years, when technology and digital formats have given a new impetus to this peculiar look.

Among them, Hardcore Henry (llya Naishuller, 2016), a film in subjective view that takes advantage of one of the prevailing perspectives of the present time: the videogames. An 
essential film to study the legacy of The Lady of the Lake under the keys of the cinema show, and to explore, also, the interactions of the cinema with a ludic universe that the spectator knows better and better. Through this, and other connected examples like Doom (Andrzej Bartkowiak, 2005), Gamer (Neveldine and Taylor, 2009) or Elephant (Gus Van Sant, 2003), we want to illuminate one of the key questions of current cinematography: What happens when you decide to film a movie from the perspective of a video game?

Keywords: Cinema, Videogame, POV, Subjective Camera, show.

El 23 de enero de 1947, la Metro-Goldwyn-Mayer anuncia la mayor "revolución técnica" desde la invención del cine sonoro': La dama del lago (Lady In The Lake), la primera película rodada en su práctica totalidad con cámara subjetiva. "Un sorprendente y atrevido nuevo método de narración" que permite al espectador sumergirse, durante una hora y cuarenta y cinco minutos, en la piel (y el punto de vista) del detective Philip Marlowe.

A pesar del atractivo de la propuesta y del apoyo de la campaña publicitaria de MGM, el film dirigido por Robert Montgomery transita sin pena in gloria entre el público y la taquilla². La crítica especializada prefiere, en cambio, arremeter contra el experimento acusándolo de errático y especialmente inadecuado para adaptar la novela homónima de Raymond Chandler (1943). No perdonan sus artificios estéticos, ni el replanteamiento de su guion adaptado (en manos del director y del guionista Steve Fisher). Mucho menos, la inserciones "explicativas" de un prólogo, un interludio y un epílogo donde la cámara gira en redondo para mostrar al protagonista (interpretado por Montgomery) dirigiéndose al espectador.

Con el paso de los años, la película va quedando relegada entre las últimas categorías de la serie negra y las listas de efemérides cinéfilas. Y aún hoy siguen incorporándose críticas desfavorables sobre el film en plataformas como Filmaffinity o Rotten Tomatoes. La mayoría de los firmantes se han visto atraídos por la anécdota, o han decidido revisitar el film por el mismo culpable que le dio y le arrebató su lucimiento: la cámara subjetiva.

Pasaría más de medio siglo hasta encontrar en la cartelera nuevos estrenos filmados enteramente con este recurso. Películas como El proyecto de la bruja de Blair (The Blair Witch Project, Eduardo Sánchez, Daniel Myrick, 1999), REC (Jaume Balagueró, 2007), Cloverfield (Matt Revees, 2008) o Enter the Void (Gaspar Noé, 2009), recuperaron la mirada subjetiva planteada por La dama del lago para ofrecer su particular revisión de géneros y experiencias cinematográficas. Los primeros, recurriendo a la subjetividad estética de los mass media y los vaivenes de la "cámara en mano", como recurso empático con el espectador (hoy acostumbrado a las tosquedades visuales de la TV e Internet).. El último, Enter the Void, empleando este recurso para desdibujar la experiencia cinematográfica tradicional y darnos una visión ácida e introspectiva de la existencia.

Un estreno reciente nos devuelve también al cine esta mirada, esta vez, desde otra de las perspectivas imperantes de la actualidad: la de los videojuegos. Hardcore Henry (2016) de llya Naishuller, se presenta como la suma visceral de la partida de juego y el metraje cinematográfico. Una excusa ideal para hormonar el legado de La dama del lago con impactantes dosis de acción y efectos especiales; pero para explorar también, las

1 Esta información publicitaria puede consultarse en el propio tráiler del film. Recuperado de: https:// www.youtube.com/watch?v=m0k5GSZ7P5E [última consulta: 30/10/2016].

2 Según IMDB, se contó con una producción estimada en 1,026,00 dólares y la recaudación no dobló la cifra: 1,812,000 dólares»; <http://www.imdb.com/title/tt0039545/business?ref_=tt_dt_bus> [consulta: 19 febrero 2017]. 
interacciones del cine con un universo lúdico que, como a nadie se le escapa, el espectador cada vez conoce mejor. Ese es, sin duda, uno de los atractivos del film y una de las preguntas clave a las que, cada vez más, se acerca la cinematografía actual: ¿Qué ocurre cuando decides filmar una película desde la perspectiva de un videojuego?

\section{PORNOGRAFÍA DE LA ACCIÓN}

Vértigo a raudales. Nos precipitamos desde el cielo abierto sobre el asfalto. Rodando entre cristales, sangre y los restos de nuestro paracaídas fallido, solo para caer, esta vez, en manos de unos matones rusos con ganas de zarandearnos. Para cuando escapamos y se ha estabilizado la mirada subjetiva del protagonista, aún nos queda otra sesión de peleas, un largo tiroteo -con una colección de armas que va, desde la ametralladora, hasta el limpiaparabrisas de un coche-, y alguna que otra imprevista explosión antes de llegar a un remanso de tregua en el guion.

Con todo, el espectador (al menos el espectador medio) ${ }^{3}$ sigue centrado y sin mareos. El uso de las cámaras Gopro, de amplio campo de visión ${ }^{4}$ y desarrolladas originalmente para acoplarse al cuerpo de los deportistas, consigue una mayor estabilidad a la hora de enfrentarse visualmente a estas proezas. Su reducido tamaño, además, permite fijarse en la cabeza del protagonista sin oscilaciones (su tecnología cuenta también con sistemas de estabilización avanzados); preparada para captar con todo detalle la coreografía de la mirada y los movimientos, desde el punto de vista subjetivo.

El resultado es mucho más estable, y por tanto menos confuso, que otros films que anteriormente ya usaron la técnica subjetiva en secuencias de acción como, por ejemplo, Días extraños de Kathryn Bigelow (1995)5. Además, la definición de la cámara (en 4K), y el gran angular proporcionado en su óptica, ofrecen una amplia apertura de toma, definida y sin distorsiones. La cámara de Hardcore Henry se adhiere a la mirada del protagonista con la estabilidad y el campo de visión necesarios para disfrutar de la experiencia, y sin dejar de prescindir de las exaltaciones dinámicas que producen nuestros movimientos reflejos. Un enfoque que recuerda, inevitablemente, a los anchos formatos de visión de los videojuegos en vista subjetiva. Especialmente, al que ofrecen los juegos de "disparo en primera persona" o first-person shooters (FPS). Exitoso subgénero de la lúdica digital donde, como ocurre en Hardcore Henry, recorremos intrincados escenarios contra hordas de enemigos, mientras solo apreciamos a ver, de nuestro cuerpo virtual, nuestras manos armadas.

Al mismo tiempo, las técnicas y el concepto visual de Hardcore Henry son también deudores de los vídeos POV (siglas de point of view). Una masa de videos, ampliamente difundida en Internet, cuyos realizadores muestran también preferencia por las cámaras Gopro (u análogas) para filmar en vista subjetiva sus propias acciones.

Si la evocación a los juegos FPS invita en Hardcore Henry a la violencia estilizada y los "espectáculos imposibles" en formato subjetivo; las reminiscencias a los vídeos POV abren,

3 En ninguna de las diversas críticas consultadas -especializadas y amateur-, se hace referencia a las turbulencias de la imagen o a las incomodidades o posibles mareos que estos frenéticos movimientos podrían provocar.

4 La cámara de gama alta, Gopro Hero4 Black, usa un "ultra gran angular" a 30, 25 y 24 fotogramas por segundo.

5 Película en la cual estos planos se rodaron en $35 \mathrm{~mm}$, con la dificultad que ello entraña en cuestiones de tamaño y soportes de cámara. 
por su lado, una puerta hacia la abundancia sensitiva a las que nos están acostumbrando los nuevos formatos audiovisuales. Nos referimos a la "ultradefinición" de las pantallas y videos en Alta Calidad (HD), con un rango dinámico de pixeles capaces de ofrecernos hasta los poros de la piel de un actor; pero, también y al mismo tiempo, a la visión epidérmica que termina germinando en el uso de las webcams, las cámaras de los teléfonos móviles u otros dispositivos conectados a la Red. Un encuadre donde todos los rostros y los objetos resultan cercanos e inmediatos; simultáneamente enfocados para observarse bajo cualquier condición ambiental (lo relevante es la transmisión).

No en vano, la etiqueta POV también es el indicativo de un género pornográfico de éxito, especializado en mostrar de forma directa, y sin apenas cortes o transiciones, la experiencia sexual desde la vista subjetiva. Un derivado del llamado porno gonzo (género que intenta involucrar al espectador directamente en la escena sexual) ${ }^{6}$, que busca intensificar la experiencia a través del factor inmersivo que proporciona esta técnica -precediendo prácticamente a la realidad virtual-, hoy mucho más factible y accesible gracias a las cámaras y a los dispositivos antes comentados.

Ante el POV de Hardcore Henry, visceral en sus espectáculos de lo sensitivo y lo inmersivo, bien podríamos hablar de una pornografía de la acción, o de una pornografía de la "hipervisualidad" (Lipovtesky, Serroy, 2009). Un producto que exhibe en la pantalla todo lo que hay que mirar, sin figuras retóricas ni dobles sentidos en su trama o forma. En otras palabras, un producto del gonzo cinematográfico: entretenimiento dopado con la tecnología necesaria para exacerbar la verborrea y el impacto audiovisual.

Como "nuevo espectador", nacido en 1983, a Naishuller estas lógicas del exceso y el impacto, provenientes de los videojuegos, la tecnología y el cine espectáculo (o películas concierto, Jullier, 2004), le vienen de fábrica. En diversas entrevistas, se declara admirador de los films de acción de los 80 como Robocop (Paul Verhoeven, 1987) o La Cosa (The Thing, John Carpenter, 1982)7, y seguidor de sagas de juegos FPS como Half Life (Valve Corporation, 1998) o Quake (id Software, 1996). Naishuller también se prodiga como director de videoclips y como músico en la banda Biting Elbows. Fue, precisamente, el videoclip en vista subjetiva, Bad Motherfucker, que dirigió en 2013 para su propia banda (y que ha pasado los 40 millones de visionados en la red) el que llamó la atención del productor Timur Bekmambetov, para proponerle un largometraje del mismo estilo (Saito, 2016) ${ }^{8}$.

Para Naishuller: "(Hardcore Henry) es una carta de amor a la música, el cine y los videojuegos" (Lindbergh, 2014). Una declaración de intenciones al ritmo frenético del videoclip y las embriagueces de los FPS que, con todo lo explicado, resultaría difícil de asimilar sin la parodia gamberra y la "insolencia rockera" que también le imprime el autor (Partearroyo, 2016). Aquí cabría citar referentes cinematográficos igualmente iconoclastas como House of the Dead (Uwe Boll, 2003), Doom (Andrzej Bartkowiak, 2005) o Gamer (Neveldine y Taylor, 2009) . E incluso films, más amables o de corte más familiar, como

6 El término alude al periodismo gonzo, género en el cual el reportero es parte de la noticia. Por analogía, la pornografía gonzo coloca al operador de la cámara directamente en la acción, hablando con los actores o siendo él uno de los actores, sin separarse de los códigos y facetas del cine pornográfico convencional.

7 (Hall, 2016).

8 Inspirado, como el mismo autor ha señalado en diversas ocasiones, en el videoclip en formato POV, Smack My Bitch Up, dirigido en 1990, por Jonas Åkerlund para el grupo, The Prodigy.

9 Sin olvidar otros films como Crank: veneno en la sangre o Crank: Alto Voltaje (Neveldine \& Taylor, 2006 y 2009).

Quaderns, 12 (2017), pp. 61-68 
Spy Kids 3D: Game Over (Robert Rodríguez, 2003) o ¡Rompe Ralph! (Wreck-It Ralph, Rich Moore, 2012).

Todos ellos hacen gala de la auto-parodia, el barroco audiovisual y el ritmo frenético de los videojuegos ${ }^{10}$, para estructurar su espectáculo y justificar buena parte de sus excesos narrativos. Aunque ninguno ha sido rodado completamente en vista subjetiva, todos cuentan con momentos notables en su metraje donde se subraya este recurso, y donde pueden anotarse las pautas de lo que supone la "mirada FPS" en el cine".

La mayor diferencia entre estos films y Hardcore Henry radica en el subrayado fetichista del videojuego, diseñado en esta ocasión para contentar al fan o al espectador connaisseur, que ha acudido a la sala de cine para seguir disfrutando de sus experiencias.

La película Doom, por ejemplo, exhibe una "mirada FPS" calcada al juego homónimo en el que se basa (Doom, id Software, 1996)12. La experiencia visual entre la adaptación cinematográfica y el juego resulta prácticamente idéntica durante el último tercio del film, con movimientos de cámara que incluso imitan la dinámica del jugador y sus reacciones frente al joystick: disparando, esquivando ataques, ocultándose en las esquinas del pasillo, etc. de la misma manera que lo hace cuando disfruta de la consola de juegos ${ }^{13}$.

En esta misma línea, la película Gamer emplea el estilo enérgico y saturado de los FPS para articular, no solo su propio espectáculo sensorial, sino también las secuencias narrativas de su argumento: un futuro distópico, en el que los más desfavorecidos, alquilan sus cuerpos para que se empleen como avatares de juego en el mundo real. Las continuadas escenas de acción, donde los personajes/avatares luchan por su vida en escenarios compactados y segmentados cual nivel de juego (un área de combate, la base del "jefe final", etc.), se van conectando durante el film por interludios de calma. De tal manera, la curvatura de eventos habitual de un juego FPS, tensión-parada-tensión (definida por niveles, misiones y dificultad), adquiere en Gamer la misma relevancia que su propia curva dramática. Esto es, un vaivén narrativo y visual, que alterna la "mirada FPS" de los planos de acción, con los planos más habituales del cine en los momentos de calma.

Donde Hardcore Henry construye su mirada subjetiva con cámaras Gopro y especialistas de cine ${ }^{14}$, Doom y Gamer, recurren a la steadicam (estabilizadores de cámara) y a los efectos digitales (CGI) para trazar su propia perspectiva subjetiva. Misma técnica de otros ejemplos

10 Juegos first-person shooter como los pertenecientes a la saga Battlefield (EA Digital Illusions, desde 2002), Call of Duty (Infinity Ward, desde 2003), Bioshock (2K Games, 2007-2015) o Far Cry (Crytek, desde 2004), entre otros.

11 No nos referimos en este artículo al ejercicio inverso, es decir, a cómo los desarrolladores de videojuegos, durante diferentes décadas, han absorbido elementos del cine para diseñar sus plataformas lúdicas. Para referirnos a este aspecto, se recomienda la lectura de Gaming: Essays on Algorithmic Culture (2006). Su autor, Alexander Galloway, dedica un capítulo completo a la deuda cinematográfica contraída por los first-person shooters donde indaga en películas como Charada (Stanley Donen, 1963) o Topaz (Alfred Hitchcock, 1969) para suscribir las modalidades de juego y visión características de estas plataformas.

12 Considerado uno de los principales precursores de los FPS, junto a juegos como Wolfenstein 3D (id Software, 1992) o los citados Half Life y Quake.

13 Una secuencia que cumple con las expectativas de los seguidores del juego ofreciendo, además, otras experiencias características de la plataforma original como: los ataques a bocajarro, los cortes con sierra eléctrica, - las riadas de sangre que inundan la pantalla. Los títulos de crédito finales del film, además, también imitan el modus operandi del circuito por los pasillos habitual en el juego.

14 A destacar, por su arduo trabajo coreográfico en el film, el especialista e intérprete "tras los ojos" del protagonista, Sharlto Copley (a quién nunca vemos el rostro). 
citados como Avalon, Spy Kids 3D: Game Over, House of the Dead ${ }^{15}$ o la misma jRompe Ralph!, esta vez -y como el resto del film- realizada enteramente con gráficos 3D. Unos films prefieren subrayar su deuda con el videojuego, incluyendo interfaces, marcadores de puntuación (HUD's $\left.{ }^{16}\right)$, etc., sobre el metraje del film. Otros se mantienen y eligen obviar estos signos, optando por una visión más próxima (en su propia ficción) a la imagen real. Se trata, al fin y al cabo, de una cuestión de estilo y presupuesto, de enfoque visual y de dinero para desarrollarlo; pero el énfasis, en la práctica totalidad, continúa cayendo en el mismo punto: explotar la experiencia inmersiva.

Llegados a este punto, cabría subrayar que la inmersión del tipo FPS no es exclusiva del cine de acción, ni se postula únicamente hacia los derroteros del cine mainstream. Este tipo de mirada también ha sido objeto de planteamientos más periféricos, o, si cabe, menos aferrados a los espectáculos y narrativas habituales del cine hollywoodiense. En su película Elephant (2003), el cineasta Gus Van Sant da buena cuenta de cómo trascender los ejercicios de estilo anteriores, empleando la mirada del videojuego sobre fenómenos más complejos, como la masacre acontecida en el instituto de Columbine en Colorado (EEUU) en 1999.

Van Sant examina este fatídico episodio desde la condición adolescente y su relación con la violencia y la cultura norteamericanas. Y para ello recurre a una reiteración estilística de largos planos secuencia en vista subjetiva que, tanto señalan la vacuidades cotidianas de los pasillos del instituto, como la afición -igualmente fría y continuada- de los asesinos por los videojuegos FPS. Estos planos se producen siguiendo un travelling espacial, que sigue los trayectos de distintos alumnos y que va moldeando, uno tras otro a lo largo del film, una temporalidad densa, deudora de la cinestesia de juegos como Doom o Half-Life, pero también de los tiempos cinematográficos expandidos de Béla Tarr o Aleksandr Sokurov (Falsetto, 2015: 87).

El cineasta recurre así a una de las propiedades intrínsecas de la lúdica digital: la pulsión dinámica que imprime la acción de juego. Lo que Manovich tilda de lógica o estética continuista (2005) y Darley de cinestesia vicaria (2002:237-44). Un modo de diseñar la secuencia en función de la dinámica específica que desprenden los juegos digitales. Van Sant emplea esta sinergia para dar forma al "elefante" que da título al film: "Un gigantesco sistema grisáceo muy opresivo e invisible, donde reside el problema". (Riambau cintado a Van Sant, 2011:165). Al mismo tiempo, para establecer un significativo paralelismo entre la mirada de los asesinos, usuarios habituales de estos videojuegos de disparos (según el film y las noticias posteriores), y la visión subjetiva de los personajes digitales que ellos manejan en la consola.

\section{PAUTAS DE LOS FPS EN LA PANTALLA DE CINE}

Cualquiera de los casos citados anteriormente podría servir a los debates que abre la perspectiva FPS en el cine. Cada uno contiene, en su crisol de facetas y estilos, las claves necesarias para desgranar la trasposición inmersiva del avatar de juego sobre la mirada

15 El director Uwe Boll, parece alcanzar el paroxismo de estas yuxtaposiciones, cuando en House of the Dead, filma una "carnicería" de más de diez minutos entre zombies y humanos, mientras inserta -sin pudorescenas directamente extraídas del juego.

16 Información permanente en la pantalla de juego que, durante la partida, muestra la puntuación, el estado y resistencia del avatar, mapas, objetos conseguidos, etc. 
cinematográfica. Hardcore Henry, tal y como se ha analizado, supone una vuelta de tuerca a las dinámicas del videojuego que films como, por ejemplo, Doom o Gamer, ya anunciaban en sus metrajes. Al mismo tiempo ninguno de ellos, puede rechazar las deudas con otros fenómenos en vista subjetiva como El proyecto de la bruja de Blair, Rec y, tras ellos, otros precursores como La dama del lago. Cabría enumerar, entonces y para finalizar, cuales son estas pautas y cualidades que los FPS imprimen en la subjetiva mirada cinematográfica:

-Primero y de forma evidente, la superposición de la vista subjetiva del juego digital sobre la vista subjetiva cinematográfica. Una incorporación que imprime la textura y el campo de visión habitual del videojuego siguiendo sus habituales particularidades: apertura, ángulo y distancia focal necesarias para que la partida se desarrolle adecuadamente (mejor visión del escenario y los enemigos, incorporación interfaces en las esquinas de la pantalla, etc.)

-En segundo lugar, el énfasis dado a la dinámica de la acción o a la estética continuista proveniente de los juegos FPS. Como este tipo de films, recogen las cinestesias características del videojuego -su pulsión por el desplazamiento ininterrumpido (esquivas, saltos, carreras, etc.)-, y las aplican sobre su metraje, ya sea como un elemento narrativo, o como una propiedad estilística.

-Cadencias que nos llevan también a la elección del plano secuencia, como principal recurso cinematográfico para imitar a los FPS. Si la película busca exaltar la sensación inmersiva del videojuego en primera persona, lo habitual es la ausencia de cortes o montajes narrativos que puedan desligar al espectador de ese momento presente de la acción.

-El empleo de la tecnología audiovisual sirve, en este aspecto, para ensalzar las condiciones del entretenimiento y las pirotecnias audiovisuales a las que nos tienen generalmente acostumbrados este tipo de films. Mejorando y estabilizando, por un lado, los planos secuencia comentados (steadicam, cámaras Gopro, etc.) y, por otro, ejerciendo de plataforma catalizadora de la experiencia inmersiva: en especial, gráficos 3D y técnicas de grabación al estilo POV, que potencian la "sensación de juego" y la experiencia sensitiva del espectador.

-A estas técnicas digitales, también le debemos la inclusión de los signos y efectismos propios de la lúdica digital. Interfaces, HUD'S, y otros símbolos digitales antes comentados que, ciertas películas, emplean para redibujar el contexto de juego sobre la pantalla de cine.

-Por último, cabría citar la comprensión o la toma de conciencia del riesgo que supone trasladar los excesos del videojuego al cine. Un factor, a priori disfuncional para el celuloide -por la propia idiosincrasia interactiva de la lúdica digital-, que lleva a muchos films a escoger entre la auto-parodia (Hardcore Henry) o entre el subrayado (Elephant) de los elementos de la lúdica digital, para paliar estos desajustes o hacerlos más accesibles al espectador.

\section{BIBLIOGRAFÍA CITADA}

DARLEY, A. [2002], Cultura Visual Digital. Espectáculo en los Medios de Comunicación, Paidós, Barcelona.

FALSETTO, M. [2015], Conversations with Gus Van Sant, Rowland \& Littlefield, Maryland, EEUU.

GALLOWAY, A. [2006], Gaming: Essays on Algorithmic Culture, Minessota Press, Minessota. HALL, J. [2016], "Hardcore Henry. Director llya Naishuller on How Making a First-Person Action Movie Is a Pain the Butt»; <http://www.slashfilm.com/hardcore-henry-ilyanaishuller-interview//2> [consulta: 24 febrero 2017]. 
JULLIER L. [2004], La Imagen Digital. De la Tecnología a la Estética, La Marca, Buenos Aires. LINDEBERGH, B. [2014], «Q\&A: Director Ilya Naishuller on the Making of 'Hardcore,' His All-First-Person POV Action Movie»; <http://grantland.com/hollywood-prospectus/ qa-director-ilya-naishuller-on-the-making-of-hardcore-his-all-first-person-pov-actionmovie/> [consulta: 10 abril 2017].

LIPOVTESKY, G. y SERROY, J. [2009], La Pantalla Global. Cultura Mediática y Cine en la Era Hipermoderna, Anagrama, Barcelona.

MANOVICH, L. [2005], El Lenguaje de los Nuevos Medios de Comunicación, Paidós Comunicación, Barcelona.

PARTEARROYO, D. [2016], Hardcore Henry; http://cinemania.elmundo.es//peliculas/ hardcore-henry/critica [consulta: 20 abril 2017].

RIAMBAU, E. [2011], Hollywood en la Era Digital. De Jurassic Park a Avatar, Cátedra, Madrid.

SAITO, S. [2016], «Interview: Ilya Naishuller on Giving Action an Attitude with Hardcore Henry»

<http://moveablefest.com/moveable_fest/2016/04/ilya-naishuller-hardcore-henry.html> [consulta: 20 abril 2017]. 